\title{
Pharmacotherapy in conjunction with lifestyle for the treatment of obesity complications, is it enough?
}

\author{
Werd Al-Najim ${ }^{1}$, Carel W. le Roux ${ }^{1,2}$ \\ ${ }^{1}$ Diabetes Complications Research Centre, Conway Institute, University College Dublin, Dublin, Ireland; ${ }^{2}$ Investigative Science, Imperial College \\ London, London, UK \\ Correspondence to: Professor Carel W. le Roux. Diabetes Complications Research Centre, Conway Institute, University College Dublin, Dublin, \\ Ireland. Email: carel.leroux@ucd.ie. \\ Provenance: This is an invited Editorial commissioned by the Section Editor Kaiping Zhang (AME College, AME Group, China). \\ Comment on: Bohula EA, Scirica BM, Inzucchi SE, et al. Effect of lorcaserin on prevention and remission of type 2 diabetes in overweight and obese \\ patients (CAMELLIA-TIMI 61): a randomised, placebo-controlled trial. Lancet 2018;392:2269-79.
}

Submitted Nov 20, 2018. Accepted for publication Nov 23, 2018.

doi: 10.21037/atm.2018.11.60

View this article at: http://dx.doi.org/10.21037/atm.2018.11.60

Type 2 diabetes and its precursor 'prediabetes' are common complications of obesity. While the rates of those diseases are reaching dangerous levels globally, effective non-invasive remission methods of the diseases are still lacking. The CAMELLIA-TIMI 61 in The Lancet shed light on the use of lorcaserin for the prevention and remission of type 2 diabetes for people with overweight and obesity (1). Lorcaserin is an FDA approved selective agonist for 5-hydroxytryptamine $2 \mathrm{C}$ serotonin receptors (5-HT2C) for the management of obesity as it reduces the symptoms of obesity (excess hunger and lack of satiety) via actions on the subcortical areas of the brain which regulate appetite $(2,3)$.

Patients with prediabetes or type 2 diabetes are asked to lose weight to control the progression of the disease. Lifestyle interventions are used as the first line therapy towards achieving moderate weight loss targets. Only 1 in 8 men and 1 in 7 women are able to lose $5 \%$ of their weight (4) with even fewer achieving the $10 \%$ weight loss target required to substantially improve type 2 diabetes (5). Once overweight or obesity occurs, the probability of attaining a normal weight with lifestyle and pharmacotherapy becomes 1 in 210 for men and 1 in 124 for women, and 1 in 1,290 for men and 1 in 677 for women with morbid obesity (4). These figures have made us aware on the complexity of treating this disease and the importance of personalizing treatments to obtain better success. However, the response to treatment is governed by biology and not external motivation. The central nervous system controls many biological parameters in the body to keep different systems at a balance including hormones, fluid, and food intake and energy expenditure. It is now accepted that body fat is also regulated by this system. The body fat set-point is still a theory, but it allows us a theoretical framework to understand the importance of adjusting treatments.

Lorcaserin results in a dose-dependent weight loss when given in conjunction to lifestyle modification (6). Bohula et al. [2018] showed that in a general population of patients with obesity, lorcaserin resulted in a net weight loss of $3.3 \mathrm{~kg}$ at 12 months, this translated to a $5 \%$ weight loss in $37.4 \%, 39.7 \%$, and $42.3 \%$ of patients with diabetes, prediabetes, and normoglycemia, respectively. Previously $5 \%$ weight loss was thought to be sufficient to reduce risks of obesity related complications. This is true for certain complications but not all. For example, as little as 5\% weight reduction can improve glycaemic control in type 2 diabetes (7), whereas to get remission of type 2 diabetes, or clinical improvement of sleep apnoea, weight loss of $10-15 \%$ may be required $(5,8)$. The rates of patients achieving $10 \%$ weight loss in Bohula et al. [2018] was 14\%, $15 \%$, and $18 \%$ of patients with diabetes, prediabetes, and normoglycemia, respectively. The higher doses of lorcaserin can result in $22.6 \%$ of patients achieving more than $10 \%$ weight loss (6) which should really become the new target.

Lorcaserin resulted in a reduction in HbA1c of $0.33 \%$ at 12 months in patients with diabetes (baseline HbAlc $53 \mathrm{mmol} / \mathrm{mol}$ or $7.0 \%$ ) and slightly lower reduction in 
those patients with prediabetes $0.09 \%$ or normoglycemia $0.08 \%$. Despite the modest reduction in HbA1c in patients with prediabetes, Bohula et al. [2018] reports that lorcaserin may be used to prevent the development of type 2 diabetes in patient with prediabetes by $19 \%$, what corresponds to treating 56 patients with prediabetes to prevent one diabetes event over 3 years, and by $23 \%$ in patients with normoglycemic (1). The authors report that improvement in HbAlc may not be weight dependent as the reduction in glycaemic parameters peak at around 3 months before the nadir in weight.

Hypoglycaemia is common in patients with diabetes taking insulin or sulfonylurea, and can cause disability or even be life threatening. There was a numerical imbalance for severe hypoglycaemia with serious complication that required hospitalization or considered life threatening or disabling in patients with diabetes taking lorcaserin [12 cases $(0.4 \%$ of all patients)] vs. placebo [ 4 cases ( $0.1 \%$ of all patients)] $(\mathrm{P}=0.054)$. The number of cases of hypoglycaemia in patients without diabetes at baseline was 9 (4 mild, 2 moderate, 2 severe, and 1 severe with serious complications) in those taking lorcaserin $v s .3$ in placebo ( 2 mild, and 1 moderate).

Lorcaserin lowered diabetes related microvascular complications by $21 \%$ in patients with diabetes (1) and lowered the rate of new onset or progression of renal impairment in patients with obesity and overweight (9).

Undesired side effects included headache, nausea, and dizziness (6), which put people at risk of stopping the treatment, particularly, if patients are not rewarded with significant reduction of the numbers on their scales early on. Initial weight loss response is a helpful indicator to identify "early responders" and "early non-responders", which may help to determine follow-up treatments or changes in approaches that may help the patient. Following up with patients closely and setting milestone targets can help determine who is responding to the treatment. For example, at 16 weeks, those who lose $5 \%$ of their weight should continue on the medication, while at 32 or 52 weeks only those who lose $10 \%$ of their weight should continue to be prescribes the medication. At each those milestone targets, if weight loss was not achieved then a different treatment should be considered, which can include a change to another diet, medication or even bariatric surgery.

Obesity cannot be cured but must be managed for life similar to how we manage all other chronic diseases such as asthma, hypertension or familial hypercholesterolemia. Successful obesity treatments point towards the subcortical brain regions where the body fat set point is regulated. Lorcaserin as a selective agonist for 5-HT2C addresses the pathology of the disease of obesity in the hypothalamus (3) by reducing the symptoms of obesity. Together with GLPanalogues and combination of naltrexone and bupropion which also address the symptoms of obesity by activating receptors in the subcortical areas of the brain, they can have beneficial effects and lower body weight set point. The next step would be to assess whether using combination pharmacological approaches plus or minus lifestyle interventions have any additive benefit, but for now we are able to offer a more personalized medicine approach for patients with obesity as we attempt to get the right treatment to the right patient at the right time.

\section{Acknowledgements}

Funding: Supported by the Irish Research Council Enterprise Partnership Scheme Postdoctoral Fellowship to Dr. Werd Al-Najim (EPSPD/2017/116), Irish Research Council Laureate grant, US-Ireland Partnership grant by Health Research Board, and Science Foundation President of Ireland Young Researcher Award to Prof Carel le Roux.

\section{Footnote}

Conflicts of Interest: CW le Roux serves on advisory boards for Novo Nordisk, Johnson \& Johnson, GI Dynamics, Fractyl, Keyron and Herbalife. W Al-Najim has no conflicts of interest to declare.

\section{References}

1. Bohula EA, Scirica BM, Inzucchi SE, et al. Effect of lorcaserin on prevention and remission of type 2 diabetes in overweight and obese patients (CAMELLIA-TIMI 61): a randomised, placebo-controlled trial. Lancet 2018;392:2269-79.

2. Thomsen WJ, Grottick AJ, Menzaghi F, et al. Lorcaserin, a novel selective human 5-hydroxytryptamine2 $\mathrm{C}$ agonist: in vitro and in vivo pharmacological characterization. $\mathrm{J}$ Pharmacol Exp Ther 2008;325:577-87.

3. Roth BL, Willins DL, Kristiansen K, et al. 5-Hydroxytryptamine2-family receptors (5-hydroxytryptamine2A, 5-hydroxytryptamine2B, 5-hydroxytryptamine2C): where structure meets function. Pharmacol Ther 1998;79:231-57.

4. Fildes A, Charlton J, Rudisill C, et al. Probability of an 
obese person attaining normal body weight: cohort study using electronic health records. Am J Public Health 2015;105:e54-9.

5. Lean ME, Leslie WS, Barnes AC, et al. Primary careled weight management for remission of type 2 diabetes (DiRECT): an open-label, cluster-randomised trial. Lancet 2018;391:541-51.

6. Fidler MC, Sanchez M, Raether B, et al. A one-year randomized trial of lorcaserin for weight loss in obese and overweight adults: the BLOSSOM trial. J Clin Endocrinol Metab 2011;96:3067-77.

Cite this article as: Al-Najim W, le Roux CW. Pharmacotherapy in conjunction with lifestyle for the treatment of obesity complications, is it enough? Ann Transl Med 2018;6(Suppl 2):S109. doi: 10.21037/atm.2018.11.60
7. Espeland M. Reduction in weight and cardiovascular disease risk factors in individuals with type 2 diabetes: one-year results of the look AHEAD trial. Diabetes Care 2007;30:1374-83.

8. Peppard PE, Young T, Palta M, et al. Longitudinal study of moderate weight change and sleep-disordered breathing. JAMA 2000;284:3015-21.

9. Scirica BM, Bohula EA, Dwyer JP, et al. Lorcaserin and Renal Outcomes in Obese and Overweight Patients in the CAMELLIA-TIMI 61 Trial. Circulation 2018. doi: 10.1161/CIRCULATIONAHA.118.038341. 